\title{
Bimanual wheelchair propulsion by people with severe hemiparesis after stroke
}

\author{
Brendan W. Smith (Corresponding Author) \\ Department of Mechanical Engineering \\ Loyola Marymount University \\ brendan.smith@1mu.edu \\ (310) 258-5433 \\ 1 LMU Dr., Los Angeles, CA 90045 \\ Diana R. Bueno \\ $\mathrm{COO}$ at Exovite Laboratorio $\mathrm{I}+\mathrm{D}$ \\ diana@exovite.com \\ Daniel K. Zondervan \\ Vice President of Flint Rehabilitation Devices, LLC \\ dzondervan@flintrehab.com \\ Luis Montano \\ Department of Computer Science and Systems Engineering \\ University of Zaragoza, Spain \\ montano@unizar.es \\ David J. Reinkensmeyer \\ Departments of Anatomy and Neurobiology, Mechanical and Aerospace Engineering, \\ Biomedical Engineering, and Physical Medicine and Rehabilitation \\ University of California, Irvine \\ dreinken@uci.edu
}




\section{Abstract (250 word max)}

Aim: Individuals who require manual wheelchairs after stroke are typically taught to ambulate with compensatory propulsion (i.e. using their nonparetic arm and foot), risking disuse of the paretic arm. We investigated whether stroke survivors can instead ambulate in a bimanual, leverdriven wheelchair that requires the paretic arm to contribute half the propulsive input.

Methods: Seventeen individuals with chronic stroke and severe hemiparesis (upper extremity Fugl-Meyer scores between 10 and 24) participated across two experiments. In the first experiment participants $(n=12)$ ambulated in straight paths. In the second experiment participants $(n=12)$ also performed turns, using an improved version of the wheelchair that incorporated handbrakes. Twelve unimpaired controls also completed the second experiment. Motion capture and EMG were used to compare biomechanics between groups.

Results: Altogether, 15 of 17 participants with stroke could ambulate $30 \mathrm{~m}$ in straight paths, and 9 of 12 could turn $1800^{\circ}$ entirely under the power of their paretic arm. Participants with stroke exhibited largely healthy biomechanics, with minimal shoulder hiking/leaning or trunk inclination. Their arm muscle EMG patterns were similar to those used by unimpaired participants, excepting delayed elbow extensor activation.

Conclusions: Individuals with severe arm impairment in the chronic stage of stroke retain sufficient strength and coordination with their paretic arm to maneuver bimanual, lever-driven wheelchairs. We suggest bimanual, lever driven propulsion should be explored in stroke rehabilitation practice as an alternative to compensatory wheelchair propulsion, as it has the potential to exercise healthy movement synergies, which may in turn help drive use-dependent motor recovery. 
Keywords - stroke, arm, rehabilitation, mobility, synergies, motor control, muscle activation, wheelchair. 


\section{Introduction}

It continues to be hypothesized that performing a greater number of practice movements during rehabilitation after stroke improves upper limb motor recovery, ${ }^{1-4}$ as predicted by the theory of use-dependent neural plasticity. ${ }^{5}$ Yet, a major challenge to both stroke patients and stroke rehabilitation providers is to successfully achieve the high number of practice movements that is theoretically needed to exploit this neural adaptive capacity ${ }^{6}$. In a sample of upper limb rehabilitation therapy sessions in the US by Lang et al., ${ }^{7}$ stroke patients performed an average of only 32 upper limb practice movements per session, an order of magnitude less than the 400-600 repetitions per session required in animal models to elicit significant neural adaptation. ${ }^{6,8,9}$ Increasing the number of practice movements is especially difficult for individuals with severe arm weakness (about $80 \%$ of subacute stroke patients) ${ }^{10}$ since it is difficult for them to perform meaningful exercises on their own. Such individuals are typically excluded from therapy paradigms such as constraint-induced movement due to generally poor outcomes, ${ }^{11,12}$ and have difficulty using equipment such as hand cycles or weight machines. ${ }^{13}$ Current robotic therapy devices are effective ${ }^{14}$ but too expensive for widespread use.

Because cost constraints limit one-on-one time with therapists, stroke patients typically spend hours each day sitting in manual wheelchairs with their arms resting on their laps or on arm rests. ${ }^{15}$ Although a key goal of early stroke rehabilitation is to help patients recover the ability to walk, stroke survivors often use manual wheelchairs during inpatient rehabilitation for seating and transportation because of initial difficulties with balance and walking. ${ }^{16-18}$ They are taught to use their less-impaired arm and leg to propel their wheelchair, a compensatory strategy that may contribute to asymmetric tone. ${ }^{19,20}$ Alternatively, they may resort to a powered wheelchair that does not provide for upper extremity training in addition to being expensive and heavy. Either 
way, failing to train the upper extremity can lead to a cycle of learned disuse and decreasing functional ability. ${ }^{21,22}$ Pushrim-activated power-assist wheelchairs (PAPAWs) help people with arm weakness better use a manual wheelchair and are successful products, ${ }^{23,24}$ but they cannot be used by people who cannot appropriately position the arms or grip and push the pushrim.

If people with hemiparesis after stroke could use their impaired arms to help propel their wheelchairs, they could perform many arm practice movements as they moved about in the normal course of their day. However, they are at present assumed to be unable to propel manual wheelchairs with their weak upper limbs. This assumption stems from the observation that they do not have enough hand function to grip and release the push rim, and because they appear to not retain enough strength or motor control to make the shoulder and elbow movements needed to propel a wheelchair forward. If a device could assist hemiparetic individuals in wheelchair propulsion, individuals could self-train their arm as they moved about, much as current manual wheelchair users train their upper extremity to a high level of fitness in the normal course of daily activity.

Moreover, there are likely other benefits of wheelchair use during subacute stroke rehabilitation therapy. According to Blower, ${ }^{16}$ using a wheelchair grants mobility to patients who otherwise might spend their time between activities relegated to a fixed chair. He argued that this may motivate patients by facilitating socialization and enhancing their senses of freedom and selfefficacy and might also exercise their spatial awareness. Although there is mixed evidence that wheelchair use by stroke patients leads to the development of asymmetric muscle tone, ${ }^{20,25} \mathrm{a}$ bimanual wheelchair that engaged both arms symmetrically could alleviate such concerns.

Here, we studied if individuals with hemiparesis after stroke could use their more-impaired arm to power one wheel of a wheelchair that utilizes Lever-Actuated Rehabilitation and Ambulation 
(LARA). A LARA-based wheelchair allows patients to propel a manual wheelchair by pumping a lever without requiring grasping and releasing of the pushrim. The lever is coupled to the wheel by a ratchet-like, one-way clutch that translates forward pumps of the lever into forward motion of the chair. In the version of the chair tested here, the lever was elastically connected to the frame of the chair, creating a mechanically resonant system. Our prior analysis shows that operating a LARAbased wheelchair at its resonant frequency could theoretically reduce the RMS torque required for sustained over-ground movement by upwards of $50 \%{ }^{26}$

Our LARA-based device is an adaptation of the Resonating Arm Exerciser (RAE), a device designed for repetitive arm movement training after stroke. ${ }^{27} \mathrm{RAE}$ consists of a lever rigidly connected to one wheel of a wheelchair, a splint for attaching the impaired arm to the lever, and elastic bands between the lever and the frame of the chair, which makes the lever system resonant. To train with RAE, the user moves the arm to create forward and backwards rocking with zero net movement. Moving at the resonant frequency of RAE rewards the user with larger arm movements.

In pilot studies with RAE, stroke patients with severe arm impairment (upper extremity Fugl-Meyer scores $<15$, out of 66 ) were able to start RAE rocking and were able to identify and move at the system's resonant frequency ${ }^{27}$ In addition, training repeatedly with RAE over three weeks reduced arm impairment. ${ }^{28}$ These findings suggested to us that such individuals might be able to ambulate overground with a lever drive wheelchair, and, based on the motivational effect of mobility, ${ }^{16}$ that such overground ambulation may help encourage arm movement practice. In addition, a previous, larger study examined the use of stereotypical, repetitive flexion and extension movements for arm rehabilitation during the acute phase of stroke and also found significant, long-term reductions in arm impairment. ${ }^{29,30}$ Thus simple, repetitive arm movementsuch as those used to propel a lever drive chair-appear to provoke beneficial motor plasticity. 
A pilot study with LARA also yielded promising results. Four participants with severe paresis after chronic stroke retained sufficient control of the shoulder and elbow to ambulate in a LARA wheelchair $30 \mathrm{~m}$ overground in straight lines. ${ }^{31} \mathrm{~A}$ second pilot study found that six participants with severe paresis after chronic stroke rapidly learned to use a version of LARA that supported increased indoor maneuverability by using a manually clutching transmission that was more challenging to learn. ${ }^{32}$

However, at the onset of this study, it remained to be rigorously determined to what extent individuals with severe hemiparesis after stroke retain sufficient motor control to maneuver lever drive chairs in indoor environments, and whether arm exercise in such chairs is a biomechanicallyappropriate activity, that could potentially contribute to rehabilitation of upper extremity motor function without exacerbating abnormal moving patterns. Here, we document two experiments to answer these questions. The first experiment extended the pilot results ${ }^{31}$ with additional subjects. The second experiment measured arm joint kinematics and muscle activity while another group of chronic stroke patients performed both straight-line and turning maneuvers with an updated version of the LARA wheelchair that included handbrakes. It was of particular interest to determine how features of arm control related to ambulation speed and whether propulsion with a lever-driven chair after stroke were associated with abnormal patterns of shoulder and arm movement, which might make it less desirable as a therapeutic adjuvant.

\section{Methods}

\section{First Experiment}

We developed a bimanual lever-driven wheelchair for use as an inpatient arm rehabilitation tool (Figure 1, top left). This wheelchair features 1 ) adjustable arm supports attached to ratcheting 
levers that support the paretic arm and position it so that the user's hand can push on the lever, 2) straps that attach the forearm and hand to the levers through the arm supports, and 3) elastic bands that limit the range of motion of the lever and make the lever resonate when pushed at $\sim 1 \mathrm{~Hz}$. Users drive the wheelchair by rhythmically flexing and extending their shoulder and elbow. The arm supports and ratcheting levers obviate the need for the user to grasp and release the push rim. The levers also give users mechanical advantage compared to directly pushing the push rim. The resonance provides further mechanical amplification of the user's arm force. ${ }^{26}$

We hypothesized that this device would allow individuals with severe arm weakness after stroke to use their paretic arm to ambulate overground in a straight line (Note: henceforth, we will refer to the more-impaired arm as the "paretic" arm, with the implicit understanding that the other, "nonparetic" arm may also exhibit some impairment or minor paresis). We tested this hypothesis by asking 12 individuals with chronic stroke (mean age $58.8 \pm 15.6$ years; mean duration since stroke $44 \pm 41$ months) to bimanually propel a first version of the LARA wheelchair. This group was equally divided by sex. Their mean upper extremity Fugl-Meyer score ranged from 10 to 23 (mean score 16.8 \pm 3.7 ) out of the maximum 66, a severe level of arm impairment. Individuals with FM scores in this range generally report never using the impaired arm for daily functional activity on a standardized arm use questionnaire, the Motor Activity Log. ${ }^{33,34}$ None of the participants had enough hand function to grasp the push rim, and none could bimanually propel a wheelchair, although half of the participants were regular users of manual wheelchairs because of their persisting gait impairment.

We asked the participants to propel the chair forward $3 \mathrm{~m}$ in a straight line 10 times, with a short rest break between each attempt. We measured the position of the wheelchair during each trial using a motion capture system (Phase Space) that tracked LEDs placed on the axis of rotation 
of each rear wheel of the wheelchair. We quantified the participants' deviation from the target straight line trajectory as the root mean square of the difference from a straight line path connecting the first and last positions of the wheelchair.

\section{Second Experiment}

Following the first experiment, we performed a second study using an improved leverdriven wheelchair. In this study, individuals with upper-extremity impairment from chronic stroke, and age-matched control subjects maneuvered in straight lines and performed $180^{\circ}$ turns. This time, we measured the arm kinematics and muscle activity. Kinematic data were recording using the same motion capture system, this time with markers placed at key points on the participant's body as well as on the wheelchair. Muscle activity data were recorded via EMG measurement of the muscles ostensibly associated with lever-driven wheelchair operation.

\section{Participants}

An age matched set of 12 participants with hemiparesis after chronic stroke (mean age $52.7 \pm 4.7$ years; mean duration since stroke $34 \pm 18$ months) and 12 unimpaired individuals (mean age $51.3 \pm 4.6$ years) participated in the second study. Both participant groups consisted of 5 males and 7 females. The stroke participants had upper-extremity Fugl Meyer (FM) scores ranging from 12 to 24 (mean score $17.6 \pm 1.3$ ) out of the maximum of 66 .

\section{Experimental protocol}

Participants performed repeated trials of two different courses: 1) a 3-m straight-line segment and 2) a 1.5-m straight-line segment followed by a the $180^{\circ}$ turn, either to the right or to the left. Stroke participants performed 10 trials of the straight course, followed by 10 trials of the $180^{\circ}$ turn course. The turn was made in the direction that positioned their paretic arm on the outside

of the turn, engaging the paretic arm while the wheel powered by the nonparetic arm remained 
stationary. Control participants performed 10 trials of the straight-line course, followed by 10 trials of the $180^{\circ}$-turn course with a left turn, followed by 10 trials of the $180^{\circ}$-turn course with a right turn.

Prior to trials, participants were seated in the LARA wheelchair. At this time, most of the stroke participants required their paretic arm and hand to be strapped to the arm support and handle. EMG electrodes and motion capture markers were then attached to their body. A full set of electrodes and markers were placed on stroke participants' paretic side and on control participants' right arm, which was the arm driving turning during the left turn trials.

Prior to the overground ambulation tasks, participants performed two 1-minute stationary practice tasks while the wheelchair's transmission was set to stationary mode and the wheel were locked in place (using the built-in, standard wheelchair brakes). In the first, they pumped only their paretic arm (right arm for controls), and in the second, they pumped both arms simultaneously.

Next came the overground ambulation tasks. For the straight-line task, participants were positioned at the beginning of the 3-m straight line course and given simple instructions about moving the levers forward and backward to move the chair forward and about the availability of the handbrake. Then the task was explained: move forward in the chair along the tape line marked on the floor until instructed to stop. Participants were given a "go" signal to begin each trial, and then a "stop" signal after they completed the course. After each trial, participants were repositioned at the starting point (by changing the chair's transmission to the neutral gear and pulling them backward), and then the next trial was started, until 10 trials were completed. Stroke participants were then asked if they were experiencing any discomfort and if they felt able to continue the experiment. 
The $180^{\circ}$-turn trials continued similarly to the straight-line trials. The new task was explained: move forward in the chair along the tape line until you reach the $180^{\circ}$ arc marked on the floor, turn the chair to follow the arc and then drive straight again until instructed to stop. Participants were allowed to begin the turn anywhere near the marked arc, as it was difficult for them to see the markings on the ground beneath them. They were instructed to 'drive straight' as soon as they completed the turn, and a second tape line, parallel to the first, served as a reference for this closing straight-line movement. The "stop" signal was given as soon as it was apparent the participant had resumed straight-line movement, usually less than $1 \mathrm{~m}$. Participants were then returned to the start point and the next trial was started. After completing all trials, electrodes and markers were removed and then the participant was helped up from the chair.

\section{Lever-driven wheelchair hardware}

The lever-driven wheelchair used for this experiment (Figure 1, top right) was fashioned after the Lever-Actuated, Resonance-Assistance (LARA) protocol and featured independent drive levers equipped with arm supports, elastic members, and additional mass to tune the resonant behavior of the system.

This chair represents a hardware improvement over the chair used during the first experiment. Each lever was also equipped with a brake handle that controlled a disc brake on the corresponding wheel. Adding this brake enabled participants to turn the chair more effectively than was possible during the first experiment. By engaging the brake on one wheel and pumping the lever of the other wheel, the chair could be made to rotate in an arc centered on the stationary wheel. Additionally, during straight-line movement, stroke participants could use the brakes to counteract their tendency to turn toward their more-impaired arm. This tendency arose from the challenge of applying equal power, simultaneously, with their paretic and nonparetic arms. 
The right and left drive lever were each coupled to their respective wheels of the chair through a one-way-bearing clutch that translated forward motion of the lever into forward motion of the respective wheel. This clutch caused backward motion of the lever to have no impact, comparable to the freewheel of a bicycle that allows the pedals to remain still (or even rotate backwards) during coasting. Additionally, each clutch could be engaged or disengaged by flipping a handle protruding from the wheel-hub of its lever. Disengaging the clutch facilitated the experimental protocol by allowing the experimenter to back up and finely position the participant as needed, still seated in the chair, between experimental trials.

Each lever was coupled to the frame of the chair through a pair of elastic elements (Figure 1, bottom), one resisting forward and the other backward lever motion, and each consisting of a pair of side-by-side tension springs (two springs were found to achieve a resonant frequency of $\sim 1$ $\mathrm{Hz}$ ). Both elastic elements were connected between the lever-hub and a shared attachment point located on the frame of the chair in front of the wheel. By positioning elastic elements both above and below the centerline between the rotational axis of the hub and that attachment point on the frame, these elastic elements were made to resist backward and forward motion of the lever respectively. The configuration had the benefit of containing the elastic elements within a smaller region than was possible with the earlier version of LARA (Figure 1, top left), which lessened interference with the motion of the lever. Additionally, the elastic elements were positioned in a precise configuration to achieve nearly linear angular elasticity over the operating range of the levers. This elasticity could be stiffened or loosened by turning a knob (Figure 1, bottom), which adjusted the horizontal position of the attachment point on the frame. This precise configuration, combined with the increased linearity of tension springs compared to the elastic bands used in the previous iteration of the chair, greatly enhanced the resonant behavior of the levers. 
This resonant behavior was augmented by the addition of steel masses added to the top and bottom of each lever. These masses increased the rotational inertia of the lever, which combined with the angular elasticity created by the tension elements, tuned the resonant frequency to $\sim 1 \mathrm{~Hz}$. The masses also nearly counterbalanced the lever. If the levers were not counterbalanced, gravity would exert a force on the lever that varied nonlinearly with lever orientation. All these factors combined to give the levers linear, resonant behavior that was consistent across participants, regardless of their arm length.

Previous work with the Resonating Arm Exerciser (RAE) suggested that stroke patients would be able to entrain their arm movement to the resonant frequency of a resonant lever, increasing the amplitude of movement of their more-impaired arm. ${ }^{27}$ Previous analytical work also suggested that resonant levers would reduce the force that participants needed to exert on the levers to ambulate in the chair at any given speed. ${ }^{26}$ The wide range of pump frequencies observed in this study, as described later, suggests that resonance may be of secondary importance to the stability imparted by the elastic elements and to their contribution to reversing the direction of the levers' motion, particularly at the end of the push phase.

Finally, each lever was equipped with an arm support that held the user's forearm as they ambulated in the chair. Each arm support featured a trough-like shape that cradled the user's forearm and supported the weight of their arm against gravity. The arm supports pivoted about their connection point to the lever, allowing consistent supporting torque to be applied to the forearm even as the angle between the forearm and the lever varied with lever orientation. The consistent supporting torque was achieved by placing a pre-loaded torsional spring between the arm support and the lever. Small variations in the relative orientations of the arm support and lever thereby cause only fractional changes in the magnitude of supporting torque applied to the forearm. 
In addition to supporting the arm against gravity, the arm supports helped prevent the forearm from bowing out as the user applied force to the lever. Straps were used to further secure the user's arm in the supports when necessary. By constraining a stroke patient's arm movement to a single degree of freedom, the arm supports were expected to simplify the control task of driving the chair and help stroke patients leverage their existing strength and coordination to propel the wheelchair.

\section{Motion capture measurement and data analysis}

Motion capture was again performed using the Phase Space motion capture system. For this experiment, however, LED Markers were placed on key positions of the participant's arm and upper-torso to measure trunk, shoulder, and elbow movement during the experiment. Markers were also placed on the wheelchair to measures its position and the orientation of the two levers. Data were collected with a sampling rate of $100 \mathrm{~Hz}$. Table 1 lists specifically where each marker was positioned on the participant's body.

Motion capture data were used to compute six kinematic features expected to describe and modulate the quality of ambulation in the chair. These features were computed for each pump of the lever, averaged across all pumps during a single trial, and then averaged across all trials for a single participant. Here, a pump is defined as one complete forward and backward movement of the corresponding lever, i.e. one period. The features were: (1) the average speed of the chair during each pump, (2) the pump frequency, computed as the reciprocal of the time duration of the pump, (3) the range of motion of the participant's torso during the pump, computed from the angle of the torso about the axis parallel to the wheelchair's axle, (4) the range of motion of elbow flexion/extension by the participant's paretic arm during the pump, (5) the total translation distance travelled by the participants' paretic-side shoulder marker during the pump, and (6) the range of motion of the lever on the participant's paretic side during the pump. 
In calculating these features, ranges of motion were computed by subtracting the minimum from the maximum values that occurred during each pump. The translation distance was calculated as Euclidean sum of the ranges of motion of each the $\mathrm{x}, \mathrm{y}$, and $\mathrm{z}$ components of the vector drawn from the participant's C7 vertebra to their shoulder in the chair's reference frame. The kinematics of features related to stroke participants' paretic side were compared to unimpaired controls' dominant hand side (all controls happened to be right handed).

Electromyogram (EMG) measurement and data analysis

EMG was performed using adhesive surface electrodes, which were placed in accordance with SENIAM (Surface EMG for Non-Invasive Assessment of Muscles) to measure 9 muscle groups: anterior deltoid, middle deltoid, posterior deltoid, biceps, triceps, wrist flexors, wrist extensors, and trapezius and pectoral major. Electrodes were positioned in a bipolar configuration and data were collected with a sampling rate of $2400 \mathrm{~Hz}$. Due to limited amplifier channels, the wrist extensors and trapezius electrodes were measured using the same channel, half of the trials measuring wrist extensors and the other half measuring trapezius. The order was randomized in a balanced fashion. The first four stroke participants and the first three control participants did not have their trapezius measured. After these participants, the alternating wrist extensors/trapezius measurement strategy was implemented, a decision informed by preliminary results of minimal wrist extensor activity.

Muscle activity was estimated from raw EMG data using established methods. ${ }^{35}$ We applied a band pass filtered with lower and upper cutoff frequencies of 20 and $400 \mathrm{~Hz}$, rectified the filtered signal, and then smoothed the result using a moving root-mean-square window with a width of $250 \mathrm{~ms}$. A lower cutoff frequency between 20 and $30 \mathrm{~Hz}$ has been shown to best remove the movement artifact. ${ }^{35}$ Analysis was performed twice, using a $20 \mathrm{~Hz}$ and a $25 \mathrm{~Hz}$ cutoff 
frequency, and very similar results acquired. The results were deemed robust to changes in cutoff frequency, and $20 \mathrm{~Hz}$ was chosen.

EMG results were primarily used to investigate the relative timing of activation of pairs of muscles. This relative timing was found for each trial, for a given pair of muscles, by finding the delay that maximized the cross-correlation of the two muscles' smoothed activity curves. To ensure that activations were compared within individual lever pumps, the magnitude of this delay was constrained to be within one half of that trial's average period. This method identifies the average timing difference between the centers of the two muscles' activations. Observing raw data revealed no clear trend in muscle activation durations, so precise initiation and termination times of activity were not estimated.

EMG results were also used to identify motor synergies, here defined as the principle components of motor coordination at the muscle level. By this definition, synergy represents the temporally and spatially correlated activations of a muscle. ${ }^{36,37}$ Each correlated muscular synergy vector $\mathbf{c}_{j}=\left(\mathrm{c}_{1 \mathrm{j}}, \ldots, \mathrm{c}_{\mathrm{mj}}\right)$ is activated by its corresponding neural signal $\boldsymbol{h}_{j}(t)$ which modulates each synergy. There are several algorithms to determine $\boldsymbol{C}$ and $\boldsymbol{H}$. In this work, we have chosen the method of Non-Negative Matrix Factorization (NNMF) since it has been shown to perform better than PCA for identifying muscle synergies from EMG data. ${ }^{38} \boldsymbol{C}$ and $\boldsymbol{H}$ were initialized with random values and iteratively updated using the multiplicative rules explained by Ajiboye and Weir ${ }^{36}$.

This method requires selecting the number of synergies to be extracted. For this work, we used the cross-validation method, ${ }^{36}$ which involves extracting the minimum number of synergies (up to the number of measured muscles, 8 in this work) such that the Variation Accounted For $(\mathrm{VAF})$ index between the original measured EMG $(\boldsymbol{V})$ and the reconstructed EMG matrix $\widehat{\boldsymbol{V}}=\boldsymbol{C}$. 
$\boldsymbol{H}$ is over 0.90 . This index is computed as $V A F=1-\mathrm{S}_{\mathrm{V} \widehat{\mathrm{V}}}^{2} / \mathrm{S}_{\mathrm{V}}^{2}$, where $\mathrm{S}_{\mathrm{V} \widehat{\mathrm{V}}}^{2}$ is the sum of the squares of the errors between original and predicted value, and $S_{V}^{2}$ is the total variance of the original EMG activation data.

The primary synergies for each participant during each task were visually compared, and common synergies were defined for each combination of group (stroke or control) and task (straight-line or $180^{\circ}$-turn) as the synergies observed most consistently across participants in that group for that task. A judgement was made about which participant represented the most typical expression of each common synergy for each group and task combination. Other participants in the same group and task were considered to express a common synergy if any of their primary synergies matched with that common synergy with a similarity index $S>0.7$. Although a fully automatic method of defining common synergies was not developed, this method was repeated with several alternative common synergies for each group until the greatest extent of similarity was obtained.

\section{Results}

Experiment 1: Can people with severe arm hemiparesis bimanually propel a lever drive chair in a straight line?

All 12 individuals in the first study had severe arm impairment (FM between 10 and 23). Yet, all were able to propel the wheelchair forward at least a total of $15 \mathrm{~m}$ over 10 trials. Some participants initially struggled with the task, preventing them from reaching the target total distance of $30 \mathrm{~m}$, but 10 of the 12 individuals successfully completed the $3-\mathrm{m}$ task by the last trial (Figure 3a). By the last trial, these 10 individuals moved in straight trajectories, while the other 2 consistently turned toward their paretic side. 
The average speed across all subjects increased significantly from the first to the last trial, reaching $0.2 \pm 0.2 \mathrm{~m} / \mathrm{s}$ on the last trial (paired t-test, $\mathrm{p}=0.014$ ). The average speed for each subject across all trials was also significantly correlated with their FM score of upper extremity impairment (Figure 3b; linear regression, $\mathrm{r}^{2}=0.44, \mathrm{p}=0.019$ ). The participant's deviation from the straight-line path decreased across trials, with the average deviation on the tenth trial being $0.05 \pm 0.05 \mathrm{~m}$, although this decrease in error was not significant. The participants performed an average of 237 arm movements during the course of the experiment (range $=[54,722])$. A video showing the participants propelling themselves with the device can be found at http://youtu.be/HzZjBIOxLzg. In a post-experiment interviews, several participants noted that achieving self-propulsion with their paretic arm was motivating and empowering, since it allowed them to use this arm in a meaningful way, often, they reported, for the first time in years.

Experiment 2: Can people with severe arm hemiparesis turn a lever drive chair with their hemiparetic arm?

In the second study, 12 individuals with stroke volunteered to propel an improved version of the wheelchair while undergoing motion capture and EMG recording; we also incorporated a turning maneuver in which participants squeezed a handbrake with their nonparetic hand and pumped the chair with their paretic hand. Seven of the participants in this second study had previously participated in the first study described above. All individuals in this study also had severe arm impairment, defined as FM score of 24 or below.

In this study, 9 of 12 participants completed the entirety of the experiment (10 straight-line trials and $10,180^{\circ}$ turn trials). The other 3 completed the 10 straight-line trials but no more than one $180^{\circ}$ turn trial. These participants took the opportunity they were offered to end the experiment at any time. One expressed that they were experiencing substantial pre-existing shoulder pain. All 
12 age-matched controls completed the full 30 trials. Equipment problems caused loss of motion capture data for one person with stroke and one age-matched control. Excluding the participants' whose data was lost, the mean age of the stroke participants was $52.5 \pm 5.2$ years $(49.4 \pm 5.8$ years for those who completed all 20 trials) and the mean age of the unimpaired controls was $53.1 \pm 4.8$ years.

Consistent with results from the first study, all participants with stroke in this experiment were able to propel themselves across $3 \mathrm{~m}$ for 10 trials (Figure 3). Thus, combining the first and second experiment, 15 of 17 participants with FM scores less than 24 demonstrated they could propel a bimanual, lever-driven wheelchair $30 \mathrm{~m}$. After propelling the chair these first $30 \mathrm{~m}, 9$ of 12 participants in the second experiment also propelled themselves across $1.5 \mathrm{~m}$ and performed a $180^{\circ}$ turn for 10 trials; these turns were driven solely by their paretic arms. In total, these participants demonstrated they could both propel the chair $45 \mathrm{~m}$ and turn in the chair a total of $1800^{\circ}$ (i.e. 5 full rotations).

Biomechanics Part 1: How did the individuals with stroke achieve lever-based propulsion?

Wheelchair speed and lever pumping behavior varied substantially between participants in the second experiment. Stroke participants were on average substantially slower than unimpaired controls, yet, in this second experiment, there was only a marginal correlation between FM score and average speed (Figure 4a; linear regression, $p=0.22$ ). The lack of significant correlation may be explained in that although the 3 slowest participants had among the lowest FM scores, the 3 fastest participants did not have distinctly high FM scores. Given that regression analysis is poorly conditioned for data sets where a few participants perform dramatically differently than the rest, it is unclear if the correlation in the first experiment (without handbrakes) and lack of correlation in 
the second experiment (with handbrakes) describes a true effect of handbrakes on operating the LARA wheelchair, or if it is simply an artifact of which participants happened to achieved especially fast ambulation during each experiment.

The questions then arise: what factors do explain how individuals with hemiparesis after chronic stroke achieve lever-based propulsion, and what explains faster or slower propulsion speeds if speed is not simply a function of impairment level. Two factors were investigated: the frequencies at which participants pumped the levers, and the ranges of motion of elbow movement they exhibited. Similar results were observed during both straight-line and $180^{\circ}$-turn trials. Results from straight-line trials are shown here. Results from $180^{\circ}$-turn trials were similar and are not shown here.

Similar wide ranges of pump frequency were observed for both stroke participants and controls (Figure 4b). Pump frequency was correlated with speed, but this relationship differed between stroke participants and controls (Figure 5b), implying that other dynamics were at play. Elbow range of motion, on the other hand, was mildly correlated with FM score, and substantially lower for stroke participants than for controls (Figure 4c). Elbow range of motion was also loosely correlated with speed (Figure 5c), suggesting a predictive relationship moderated by other factors. However, combining these two factors produced a much more predictive relationship, specifically the product of pump frequency and elbow range of motion was highly correlated with speed (Figure 5d). Of note, elbow range of motion and pump frequency were themselves not correlated (Figure 5e). These results imply that two uncorrelated motor capacities determine an individual's ambulation speed: 1) their ability to quickly activate and deactivate their arm muscles and 2) their ability to extend the elbow through a wide active range of motion. 
We suspected that some participants might have achieved faster speeds using abnormal compensatory movements, such as trunk inclination or shoulder leaning or hiking. On average, stroke participants showed no increased trunk motion over controls; on an individual basis, however, the three slowest participants exhibited larger trunk motion (Figure 4d). This suggests that compensation by trunk inclination is a means by which slower participants achieved movement, but most participants did not employ this compensation. Amplitude of shoulder translation, which would be expected to capture leaning or hiking, differed minimally between stroke participants and controls, and was uncorrelated with FM score (Figure 4e). Nor was greater shoulder movement observed for slower participants. This suggests that lever-based propulsion does not encourage abnormal shoulder movement in individuals with hemiparesis after chronic stroke, even for those for whom propulsion is difficult.

Biomechanics Part 2: How does stroke affect the muscle activation patterns used during lever-based propulsion?

In general, both chronic stroke and control participants exhibited a cascade of muscle activation during only the push phase of lever-based propulsion, and minimal activation during the recovery phase when the movement was assisted by the return action of the spring attached to the lever (Figure 6). This differs from the substantial muscle activity observed during both push and recovery phase during push-rim wheelchair propulsion. ${ }^{39,40}$ This finding implies that LARA reduces in the complexity of operating manual wheelchair. This raises the questions of how the timing of these cascading muscle pulses compares between stroke participants and controls and whether this might help explain differences in their propulsion speed. We also investigated the occurrence of common muscle activation synergies across participants. 
The relative activation timing between the triceps and other muscles best differentiated the activation patterns of stroke and control participants (Figure 7a). For controls participants, the activity of the triceps significantly preceded that of most other muscles (t-test, $\mathrm{p}$ values shown in Table 2 "Controls"), suggesting they initiated lever movement by activating the triceps. For chronic stroke participants, activation of the triceps did not significantly precede that of any other muscle (t-test, $\mathrm{p}$ values shown in Table 2 "Stroke"). Comparing these relative timings between stroke participants and controls, it was found that the timings of all muscles except the anterior deltoid were significantly different between groups (paired t-test, nonequal variance, $\mathrm{p}$ values shown in Table 2 "Difference"). This comparison supports that a significant shift in timing of the triceps occurs following stroke, and that it no longer leads the other muscles.

The relative timing of the biceps and triceps was investigated as a predictor of participants' propulsion speed, based on our finding that amplitude of elbow movement was correlated with participants' propulsion speed. Elbow extension ostensibly requires activating the triceps without interference of the biceps. Our observation that the triceps no longer leads the biceps after stroke might help explain chronic stroke participants' reduced elbow extension relative to control participants. To elucidate this connection, each participant's bicep activation timing was compared to their elbow amplitude (Figure 7b). A marginally significant trend was found between biceps timing and elbow amplitude for stroke participants (linear regression, $\mathrm{R} 2=?, \mathrm{p}=0.052$ ). A nonsignificant correlation existed for control participants (linear regression, $p=0.15$ ). Extrapolating this trend would suggest that someone with hemiparesis after chronic stroke could increase their range of elbow movement, and their propulsion speed by learning to activate their triceps preemptively of their biceps and other arm muscles. 
Along with delayed triceps activation, stroke participants appear to have exhibited early trapezius activation (not shown directly, but implied by Figure 7a). Using a similar analysis as described for the triceps, the trapezius was found to significantly lead the pectoralis major (t-test, $\mathrm{p}=0.030)$ and the anterior deltoid (t-test, $\mathrm{p}=0.030)$ for chronic stroke participants. This may suggest that stroke participants compensate for their impairment by preempting their arm movement with positioning or bracing of the shoulder, even if kinematic data did not show increased amplitude of shoulder movement.

EMG synergy analysis suggested largely similar muscle activation patterns across the two groups, with some discrepancy in the expression of synergies involving the triceps. For each participant, two primary synergies on average described more than the $90 \%$ of their variability in muscle activation in each task conditions. The synergies of each participant were visually compared, and two common synergies (Figure 7c) were defined for each combination of group (stroke or control) and task (straight-line or $180^{\circ}$-turn). For those who expressed them, these common synergies were remarkably similar between stroke participants and controls during both the straight-line and $180^{\circ}$-turn trials (Figure $7 \mathrm{c}$ ). Overall, these common synergies were expressed with $52 \%$ consistency across stroke participants (i.e. as one of their two primary synergies) and with $63 \%$ consistency across controls (Table 3 ). This suggests that the most common muscle activation patterns were largely shared by stroke participants and controls, but that substantial variation in these patterns existed within both groups. The common synergies that most involved the triceps ( $w_{1}$ for straight-line trials and $w_{2}$ for $180^{\circ}$-turn trials), were also expressed with $52 \%$ consistency across stroke participants, but with $79 \%$ consistency across controls. That is, triceps activation was generally synergized with the same muscles for controls. In contrast, only about 
half of stroke participants activated their triceps within any such shared such synergy. That synergy did, however, closely resemble the triceps activation synergy expressed by controls.

Intrinsic Motivation: Are individuals with stroke motivated by their performance in lever-driven wheelchairs?

All participants answered a 10-question survey with relevant questions drawn from the Intrinsic Motivation Inventory (IMI), which has been used in studies of robot-aided arm rehabilitation. ${ }^{41}$ Questions related to motivation, effort, and perceived competence (Table 4). All questions prompted a numeric response between 1 for "strong disagreement" and 7 for "strong agreement". Perhaps not surprisingly, stroke participants believed that practicing longer with the wheelchair would be more beneficial to them than did control participants (paired t-test, $\mathrm{p}=$ 0.0043). They also believed that ambulating in the chair required more effort than did control participants (paired t-test, 0.025). No significant difference was found between the responses of stroke and control participants on any of the remaining questions. Neither group felt particularly anxious or bored during the experiment. Both groups indicated that they wanted to do the activity, possibly consistent with the results that both groups found the task fun and felt that their participation was important. Both groups were satisfied with their performance despite being somewhat less confident that they were good at the activity. Both groups shared a similar level of confidence that they became competent at the activity during this session. Surprisingly stroke participants did not feel significantly less competent at ambulating in the chair than did control participants. 


\section{Discussion}

The assumption that individuals with severe arm weakness after stroke cannot bimanually propel themselves in a manual wheelchair is incorrect. Here, we found that a total of 15 of 17 participants with stroke (10 of 12 from the first experiment, and all 5 new participants from the second) propelled a manual, lever-driven wheelchair 30 to $45 \mathrm{~m}$ in a straight line, demonstrating their ability to coordinate the movement of their arms to move in a straight line. Using a handbrake during the second experiment, 9 of 12 stroke participants completed ten $180^{\circ}$ turns (for a total of $1800^{\circ}$ ) completely under the power of the paretic arm. Thus, with appropriate mechanical adaptations, even individuals with otherwise little functional use of the arm generated enough force in the required spatiotemporal pattern to propel and turn a lever drive chair. This confirms our preliminary findings that chronic stroke survivors retain this capacity, ${ }^{31,32}$ but in a larger sample. Moreover, while ambulating during this study, participants regularly performed between 400-600 arm movements during each 1-hour session. This dose of exercise exceeds that even of recent attempts to provide a high dose of movement practice in stroke rehabilitations. ${ }^{2}$ It remains to be seen if this sort of arm motion is therapeutically meaningful, but this study yields some promising results about this possibility, coupled with previous studies on the therapeutic effectiveness of simple, repetitive arm movement REFS. We discuss now several features of the lever-driven propulsion, the biomechanics of the arm and trunk, and then future directions for research.

\section{Features of lever-driven wheelchair ambulation}

The stroke participants moved at about half the speed of unimpaired controls during straight line movement and rotated the chair at about one-third the speed of controls during turns. While their turning was slower generally, the faster half of stroke participants moved in straight lines at about the same speed as the slower half of controls. Together these results indicate that 
even people with substantial upper extremity impairment can achieve meaningful ambulation speeds for straight line and turning movements in a lever-driven wheelchair.

Participants' abilities to turn, as well as to navigate in straight lines, were facilitated by the availability of the handbrake in the second experiment. Participants controlled the handbrake with their nonparetic hand (a handbrake was present on both sides of the chair, but participants either lacked the ability, or were disinclined, to use the brake on their paretic side). Braking facilitated straight line movement by allowing participants to voluntarily impede propulsion caused by their nonparetic arm, which they tended to move with greater amplitude than their paretic arm. Without handbrakes (i.e. in the first experiment), 10 of 12 participants were able to ambulate in straight lines, but this required them to regulate this imbalance between their arms, often by alternating many small pumps of the paretic-side lever with fewer larger pumps of the nonparetic-side lever. Adding the handbrake appears to have circumvented participants' use of this strategy, as seen in the predominately synchronized movement of the two levers.

Participants were also intrinsically motivated by the movement speeds achieved in this study, reporting that the task was motivating, enjoyable, and engaging. Participants with chronic stroke also reported similar confidence in their ability to use the chair as unimpaired controls, and frequently expressed excitement and satisfaction for what they could achieve with their paretic side.

\section{Biomechanics of lever-driven wheelchair ambulation}

Participants exhibited arm kinematics and muscle activity that imply that ambulating in a lever-driven chair would be meaningful to their upper extremity rehabilitation. Speed in the chair was strongly correlated with the product of two kinematic features, amplitude of elbow movement and pump frequency. These features, in turn, were found to be uncorrelated. This implies that two 
orthogonal predictors of an individual's ability to ambulate in the chair are 1) their ability to alternate between activating and deactivating their muscles, and 2) their ability to extend their elbow. In this study, we observed that stroke participants and controls, who ambulated at similar speeds, did so with different combinations of these capabilities. Stroke participants employed smaller elbow amplitudes and faster pump frequencies to achieve the same speed as slower controls. In any case, if driving a LARA wheelchair promotes these two features, then one might expect it to be beneficial for relearning arm movement, as alternating muscle activity and elbow extension are essential for performing functional activities with the arm.

Kinematic analysis alleviated a major concern of this study: whether chronic stroke patients ambulate in lever-driven wheelchairs via abnormal compensatory movements of the trunk and shoulder. Such compensation might limit the therapeutic effect of lever-driven chairs, since, then, users might simply be expected to get better at compensation, rather than arm movement. ${ }^{42}$ Across participants with chronic stroke, amplitude of shoulder motion was within the distribution of unimpaired participants'. Trunk motion was elevated only for the slowest $25 \%$ of these participants. These results show that viable ambulation speed was achieved largely without compensatory trunk or shoulder movement. This is an encouraging result also given the potential harmful orthopedic effects of applying large forces around abnormal joint orientations. ${ }^{43}$ This result was somewhat surprising given that individuals with severe stroke typically exhibit trunk compensation while practicing reaching movements directly. ${ }^{43}$ Certainly, a stroke patient's clinician would need to assess the quality of their movement, but this result supports the likelihood that propelling lever-driven wheelchairs may be an effective, biomechanically healthy means of arm movement practice, a hypothesis that warrants clinical evaluation. 
EMG analysis found that participants with stroke expressed similar patterns of muscle activation as unimpaired controls, namely a cascade of activations during the push phase of ambulation. Similar muscle synergies were observed for both groups, with the exception that the common synergy involving the triceps was less often expressed by participants with chronic stroke. This echoed the primary difference observed in muscle activation timing: that unimpaired participants generally led their muscle activation cascades with triceps activation, whereas participants with chronic stroke generally led with trapezius activation, and activated their triceps later at roughly the same time as they activated their biceps.

Delayed timing and reduced synergy of triceps activation would explain the reduced amplitude of elbow motion by participants with chronic stroke, and in turn their reduced propulsion speeds. Coordinating activation of the opposed triceps and biceps is known to be necessary for achieving larger ranges of motion with the elbow. ${ }^{44}$ As such, although triceps are not a primary source of power during traditional manual wheelchair propulsion, the triceps is important for maintaining the compliance of the elbow and coordinating its extension with the delivery of power from the deltoids and pectoralis major..$^{39,40,45}$ This is akin to the triceps' role during reaching tasks where the triceps produces relatively minimal force, but must be coordinated with the shoulder muscles and biceps to effectively control the position of the hand. ${ }^{46,47}$

Even though earlier trapezius activation by participants with chronic stroke appears not to caused abnormal shoulder movements, such as leaning or hiking, it still likely impeded leverdriven propulsion, and may have supported compensation by trunk inclination for the slowest $25 \%$ of participants. These participants likely retained some volitional control of their trapezius, consistent with the proximal to distal gradient in impairment following stroke. ${ }^{48}$ It is possible that by stabilizing the shoulder, they could then apply power with abdominal and other lower trunk 
muscles, which were not measured here, but which has been reported during demanding manual wheelchair operation. ${ }^{49}$ However, if preemptive activation of the trapezius was part of a compensatory strategy, it was minimally evident for the faster participants. Early activation of the trapezius might alternatively indicate unintentional activation while seeking to activate the anterior and middle deltoids, pectoralis major, and triceps, which are the primary muscles engaged during manual wheelchair operation by individuals with unimpaired arm function. ${ }^{40}$ Motor control is understood to be largely impeded by imbalances in the strength of agonist-antagonist pairs created by stroke, and by the increased involvement of muscles secondary to arm movement. ${ }^{47}$ Such imbalances and unintentional activations likely explain the reduced speed of the participants who otherwise expressed largely normal biomechanics.

\section{Future Directions}

Based on these results, we suggest the exploration of lever-driven wheelchairs as a potential paradigm shift in the way rehabilitation and mobility needs are addressed during chronic, and perhaps even acute stroke rehabilitation. Use of lever-driven wheelchairs could immediately increase the number of arm practice movements that chronic stroke patients perform in outpatient and community therapy settings, alleviating paretic arm disuse, and solving a longstanding problem in rehabilitation practice.

This technology might also be integrated into current inpatient clinical practice without interfering with times reserved for conventional therapy. Instead, lever-driven wheelchairs could potentially replace a patient's traditional manual wheelchair, allowing them to exercise by moving about the clinic. Rather than be pushed by a caregiver to the cafeteria or their therapy appointments, patients could then drive themselves, increasing their arm movement practice by hundreds of movements each day and enhancing their sense of self-efficacy in the process. A lever-driven 
wheelchair might even provide this independent mobility without the drawbacks associated with the compensatory good-arm good-leg strategy currently taught for wheelchair propulsion after stroke (e.g. developing asymmetric posture, learned disuse of the paretic limbs ${ }^{20}$ ). Other drawbacks of lever-driven wheelchairs such as their added weight and width have limited their acceptance for community use, but we propose that clinical use would circumvent these problems. For clinical use, a chair need not be light enough to load into a vehicle trunk nor narrow enough to pass through standard doors or hallways. Nor need it be highly maneuverable, since clinic hallways are straight, corners are broad, and speeds are low.

The next step in evaluating the rehabilitation approach we propose here is to conduct a randomized controlled trial of the use of lever-driven wheelchairs for upper limb therapy. A study is underway in which newly admitted stroke inpatients are randomly assigned either a traditional wheelchair or a lever-driven wheelchair. Those that receive the lever-driven chair use it to move about the hospital, currently under the supervision of clinicians, thus increasing their arm movement practice. The efficacy of the stationary mode is also being explored, and we have developed therapy-oriented computer games that patients can play in their rooms by moving the levers. ${ }^{26}$ Note, such stationary practice could be a meaningful stepping stone to training overground ambulation, as some participants in this study who did not exhibit healthy kinematic synergies during overground ambulation did exhibit similar synergies as unimpaired individuals during stationary practice.

\section{Clinical Messages}

- Severe arm impairment arising after stroke does not generally eliminate the motor dexterity needed to bimanually propel a manual wheelchair, if the wheelchair is modified to remove the requirement to grasp and release the push rim. 
- Such exercise appears a good candidate to facilitate rehabilitation outcomes because it depends on alternating muscle activity and improving elbow extension.

- Such wheelchair propulsion involves largely normal biomechanics; shoulder hiking and leaning are absent and trunk inclination is rare.

\section{Acknowledgements}

The authors thank Justin Rowe for his assistance writing the motion capture software and Vicky Chan for recruiting and coordinating all experimental participants. Research supported by Machines Assisting Recovery from Stroke and Spinal Cord Injury for Reintegration into Society (MARS3), the National Institute of Disability and Rehabilitation Research Rehabilitation Engineering Research Center on Rehabilitation Robotics, H133E120010, by UC Irvine Institute for Clinical and Translational Science, and by ADD Diana's funding.

\section{Author Contributions}

Order for each contribution is alphabetical. Reinkensmeyer DJ, Smith BW, and Zondervan DK, developed the lever-driven wheelchair. Bueno DR, Smith BW and Zondervan DK conducted experiments. Bueno DR, Smith BW, and Zondervan DK analyzed experimental data. All authors interpreted experimental data. Smith BW compiled the manuscript and prepared it for submission. Bueno DR, Smith BW, and Zondervan DK contributed written material. Reinkensmeyer DJ, Smith BW, and Zondervan DK revised the manuscript. All authors read and approved the manuscript in its submitted form. 


\section{Conflict of Interest}

Daniel Zondervan and David Reinkensmeyer are co-founders of and hold equity in Flint Rehabilitation Devices, a company that is commercializing rehabilitation technology. Daniel Zondervan is currently employed at Flint, and David Reinkensmeyer has received payment for consulting from Flint. David Reinkensmeyer holds equity in Hocoma, a manufacturer of rehabilitation technology. The terms of David Reinkensmeyer's interests have been reviewed by the U.C. Irvine Conflict of Interest committee. 


\section{References}

1. Lohse KR, Lang CE, Boyd LA. Is more better? Using meta-data to explore dose-response relationships in stroke rehabilitation. Stroke 2014; 45: 2053-2058.

2. Lang CE, Strube MJ, Bland MD, et al. Dose response of task-specific upper limb training in people at least 6 months poststroke: A phase II, single-blind, randomized, controlled trial. Ann Neurol 2016; 80: 342-354.

3. Bell JA, Wolke ML, Ortez RC, et al. Training intensity impacts motor rehabilitation efficacy following unilateral ischemic insult of the sensorimotor cortex in C57BL/6 mice. Neurorehabil Neural Repair 2015; 29: 590-598.

4. French B, Thomas LH, Leathley MJ, et al. Repetitive task training for improving functional ability after stroke BT - Cochrane Database Syst Rev. Cochrane Database Syst Rev. Epub ahead of print 2016. DOI: 10.1002/14651858.CD006073.pub3.www.cochranelibrary.com.

5. Sawaki L. Use-dependent plasticity of the human motor cortex in health and disease. IEEE Eng Med Biol Mag 2005; 24: 36-9.

6. Jeffers MS, Karthikeyan S, Gomez-Smith M, et al. Does Stroke Rehabilitation Really Matter? Part B: An Algorithm for Prescribing an Effective Intensity of Rehabilitation. Neurorehabil Neural Repair 2018; 32: 73-83.

7. Lang CE, Macdonald JR, Reisman DS, et al. Observation of amounts of movement practice provided during stroke rehabilitation. Arch Phys Med Rehabil 2009; 90: 1692-8.

8. Kleim JA, Barbay S, Nudo RJ. Functional Reorganization of the Rat Motor Cortex Following Motor Skill Learning. J Neurophysiol 1998; 80: 3321-3325.

9. Birkenmeier RL, Prager EM, Lang CE. Translating animal doses of task-specific training to people with chronic stroke in 1-hour therapy sessions: A proof-of-concept study. 
Neurorehabil Neural Repair 2010; 24: 620-635.

10. Langhorne P, Coupar F, Pollock A. Motor recovery after stroke: a systematic review. Lancet Neurol 2009; 8: 741-754.

11. Taub E, Uswatte G, King DK, et al. A placebo-controlled trial of constraint-induced movement therapy for upper extremity after stroke. Stroke 2006; 37: 1045-1049.

12. Wolf SL, Winstein CJ, Miller JP, et al. Effect of Constraint-Induced Movement. 2006; 296: 2095-2104.

13. Zehr EP, Loadman PM, Hundza SR. Neural control of rhythmic arm cycling after stroke. $J$ Neurophysiol 2012; 108: 891-905.

14. Mehrholz J, Pohl M, Platz T, et al. Electromechanical and robot-assisted arm training for improving activities of daily living, arm function, and arm muscle strength after stroke. Cochrane Database Syst Rev. Epub ahead of print 3 September 2018. DOI: 10.1002/14651858.CD006876.pub5.

15. Jette DU, Latham NK, Smout RJ, et al. Physical therapy interventions for patients with stroke in inpatient rehabilitation facilities. Phys Ther 2005; 85: 238-48.

16. Blower P. The advantages of the early use of wheelchairs in the treatment of hemiplegia. Clin Rehabil 1988; 2: 323-325.

17. Christina M. Mumma. Perceived Losses Following Stroke. Rehabil Nurs 1986; 11: 19-24.

18. Barker DJ, Reid D, Cott C. Acceptance and meanings of wheelchair use in senior stroke survivors. Am J Occup Ther 2004; 58: 221-230.

19. Barrett JA, Watkins C, Plant R, et al. The COSTAR wheelchair study: a two-centre pilot study of self-propulsion in a wheelchair in early stroke rehabilitation. Collaborative Stroke Audit and Research. Clin Rehabil 2001; 15: 32-41. 
20. Ashburn A, Lynch M. Disadvantages of the early use of wheelchairs in the treatment of hemiplegia. Clin Rehabil 1988; 2: 327-331.

21. Cooper R, Quatrano L, Axelson P, et al. Research on physical activity and health among people with disabilities: a consensus statement. $J$ Rehab Res Dev 1999; 36: 142-154.

22. Schweighofer N, Han CE, Wolf SL, et al. A functional threshold for long-term use of hand and arm function can be determined: predictions from a computational model and supporting data from the Extremity Constraint-Induced Therapy Evaluation (EXCITE) Trial. Phys Ther 2009; 89: 1327-1336.

23. Cooper RA, Fitzgerald SG, Boninger ML, et al. Evaluation of a pushrim-activated, powerassisted wheelchair. Arch Phys Med Rehabil 2001; 82: 702-8.

24. Nash MS, Koppens D, van Haaren M, et al. Power-Assisted Wheels Ease Energy Costs and Perceptual Responses to Wheelchair Propulsion in Persons With Shoulder Pain and Spinal Cord Injury. Arch Phys Med Rehabil 2008; 89: 2080-2085.

25. Pomeroy VM, Mickelborough J, Hill E, et al. A hypothesis: self-propulsion in a wheelchair early after stroke might not be harmful. Clin Rehabil 2003; 17: 174-80.

26. Zondervan DK, Smith B, Reinkensmeyer DJ. Lever-actuated resonance assistance (LARA): A wheelchair-based method for upper extremity therapy and overground ambulation for people with severe arm impairment. In: IEEE International Conference on Rehabilitation Robotics. 2013.

27. Zondervan DK, Palafox L, Hernandez J, et al. The Resonating Arm Exerciser: design and pilot testing of a mechanically passive rehabilitation device that mimics robotic active assistance. J Neuroengineering Rehabil 2013; 10: 39.

28. Zondervan DK, Augsburger R, Bodenhoefer B, et al. Machine-based, self-guided home 
therapy for individuals with severe arm impairment after stroke: A randomized controlled trial. Neurorehabil Neural Repair 2015; 29: 395-406.

29. Feys HM, De Weerdt WJ, Selz BE, et al. Effect of a therapeutic intervention for the hemiplegic upper limb in the acute phase after stroke: a single-blind, randomized, controlled multicenter trial. Stroke 1998; 29: 785-792.

30. Feys H, De Weerdt W, Verbeke G, et al. Early and repetitive stimulation of the arm can substantially improve the long-term outcome after stroke: a 5-year follow-up study of a randomized trial. Stroke 2004; 35: 924-9.

31. Smith BW, Zondervan DK, Lord TJ, et al. Feasibility of a bimanual, lever-driven wheelchair for people with severe arm impairment after stroke. Conf Proc . Annu Int Conf IEEE Eng Med Biol Soc IEEE Eng Med Biol Soc Annu Conf 2014; 2014: 5292-5295.

32. Sarigul-Klijn Y, Lobo-Prat J, Smith BW, et al. There is plenty of room for motor learning at the bottom of the Fugl-Meyer: Acquisition of a novel bimanual wheelchair skill after chronic stroke using an unmasking technology. IEEE Int Conf Rehabil Robot 2017; 50-55.

33. Stewart JC, Cramer SC. Patient-reported measures provide unique insights into motor function after stroke. Stroke 2013; 44: 1111-6.

34. van der Lee JH, Beckerman H, Knol DL, et al. Clinimetric properties of the Motor Activity Log for the assessment of arm use in hemiparetic patients. Stroke 2004; 35: 1404-1410.

35. De Luca CJ, Donald Gilmore L, Kuznetsov M, et al. Filtering the surface EMG signal: Movement artifact and baseline noise contamination. J Biomech 2010; 43: 1573-1579.

36. Ajiboye AB, Weir RF. Muscle synergies as a predictive framework for the EMG patterns of new hand postures. $J$ Neural Eng; 6. Epub ahead of print 2009. DOI: 10.1088/17412560/6/3/036004. 
37. Safavynia S, Torres-Oviedo G, Ting L. Muscle Synergies: Implications for Clinical Evaluation and Rehabilitation of Movement. Top Spinal Cord Inj Rehabil 2011; 17: 16-24.

38. Tresch MC, Cheung VCK, D'Avella A. Matrix Factorization Algorithms for the Identification of Muscle Synergies: Evaluation on Simulated and Experimental Data Sets. J Neurophysiol 2006; 95: 2199-2212.

39. Schantz P, Björkman P, Sandberg M, et al. Movement and muscle activity pattern in wheelchair ambulation by persons with para-and tetraplegia. Scand J Rehabil Med 1999; 31: $67-76$.

40. Chow JW, Millikan TA, Carlton LG, et al. Kinematic and Electromyographic Analysis of Wheelchair Propulsion on Ramps of Different Slopes for Young Men With Paraplegia. Arch Phys Med Rehabil 2009; 90: 271-278.

41. Novak D, Nagle A, Keller U, et al. Increasing motivation in robot-aided arm rehabilitation with competitive and cooperative gameplay. J Neuroeng Rehabil 2014; 11: 64.

42. Michaelsen SM, Dannenbaum R, Levin MF. Task-specific training with trunk restraint on arm recovery in stroke: Randomized control trial. Stroke 2006; 37: 186-192.

43. Cirstea MC, Levin MF. Compensatory strategies for reaching in stroke. Brain 2000; 123: 940-953.

44. Barker RN, Brauer S, Carson R. Training-induced changes in the pattern of triceps to biceps activation during reaching tasks after chronic and severe stroke. Exp Brain Res 2009; 196: 483-496.

45. Veeger HEJ, Van Der Woude LH V, Rozendal RH. Load on the Upper Extremity in Manual Wheelchair Propulsion. J Electromyography Kinesiol 1991; I: 270-280.

46. Buneo CA, Soechting JF, Flanders M. Muscle activation patterns for reaching: the 
representation of distance and time. J Neurophysiol 1994; 71: 1546-1558.

47. Lum PS, Burgar CG, Shor PC. Evidence for strength imbalances as a significant contributor to abnormal synergies in hemiparetic subjects. Muscle and Nerve 2003; 27: 211-221.

48. Beebe JA, Lang CE. Absence of a proximal to distal gradient of motor deficits in the upper extremity early after stroke. Clin Neurophysiol 2008; 119: 2074-2085.

49. Yang YS, Koontz AM, Triolo RJ, et al. Surface electromyography activity of trunk muscles during wheelchair propulsion. Clin Biomech 2006; 21: 1032-1041. 
Table 1. Motion capture marker positions.

\begin{tabular}{ll}
\hline MARKER & DESCRIPTION \\
\hline C7 & Spinous process of the 7th cervical vertebrae \\
\hline Clavicle & Jugular Notch where the clavicles meet the sternum \\
\hline Shoulder & Acromio-clavicular joint \\
\hline Upper arm & Equidistant between the elbow and shoulder markers. \\
\hline Lateral elbow & Lateral epicondyle approximating elbow joint axis \\
\hline Medial elbow & Medial epicondyle approximating elbow joint axis \\
\hline Forearm & Equidistant between the wrist and elbow markers. \\
\hline Radius & Wrist bar thumb side \\
\hline Ulna & Wrist bar pinkie side \\
\hline Hand & Middle of back of the hand \\
\hline
\end{tabular}


Table 2. P-values for muscle activation timing relative to triceps.

\begin{tabular}{lccc}
\hline Muscle & Stroke & Controls & Difference \\
\hline Trapezius & 0.1348 & 0.0822 & $\mathbf{0 . 0 1 9 3}$ \\
\hline Pectoralis major & 0.8405 & $\mathbf{0 . 0 0 5 9}$ & $\mathbf{0 . 0 3 0 6}$ \\
\hline Posterior deltoid & 0.2855 & 0.0650 & $\mathbf{0 . 0 3 0 5}$ \\
\hline Middle deltoid & 0.1607 & $\mathbf{0 . 0 0 2 5}$ & $\mathbf{0 . 0 0 5 6}$ \\
\hline Anterior deltoid & 0.7712 & $\mathbf{0 . 0 2 2 7}$ & 0.2410 \\
\hline Biceps & 0.2462 & $\mathbf{0 . 0 0 8 8}$ & $\mathbf{0 . 0 0 3 3}$ \\
\hline Wrist extensors & 0.5800 & $\mathbf{0 . 0 0 8 1}$ & $\mathbf{0 . 0 0 6 9}$ \\
Wrist flexors & 0.9363 & $\mathbf{0 . 0 0 3 5}$ & $\mathbf{0 . 0 0 6 5}$ \\
\hline
\end{tabular}

Bold indicates $\mathrm{p}<0.05$. 
Table 3. Expression of the first two common kinetic synergies across participants.

\begin{tabular}{|c|c|c|c|c|}
\hline \multirow{2}{*}{$\begin{array}{c}\text { Control } \\
\text { Participants }\end{array}$} & \multicolumn{2}{|c|}{ Line } & \multicolumn{2}{|c|}{ Turn } \\
\hline & $w_{1}$ & $w_{2}$ & $w_{1}$ & $w_{2}$ \\
\hline S1 & $\mathrm{C}_{1}$ & $\mathrm{C}_{2}$ & $\mathrm{C}_{1}$ & $\mathrm{C}_{2}$ \\
\hline S2 & $\mathrm{C}_{1}$ & $\mathrm{C}_{2}$ & $\mathrm{C}_{1}$ & $\mathrm{C}_{2}$ \\
\hline S3 & & $\mathrm{C}_{2}$ & $\mathrm{C}_{2}$ & \\
\hline S4 & & $\mathrm{C}_{2}$ & & $\mathrm{C}_{2}$ \\
\hline S5 & $\mathrm{C}_{1}$ & & & $\mathrm{C}_{2}$ \\
\hline S6 & & $\mathrm{C}_{2}$ & $\mathrm{C}_{1}$ & $\mathrm{C}_{2}$ \\
\hline S7 & $\mathrm{C}_{2}$ & & & $\mathrm{C}_{2}$ \\
\hline S8 & & $\mathrm{C}_{2}$ & $\mathrm{C}_{1}$ & $\mathrm{C}_{2}$ \\
\hline S9 & $\mathrm{C}_{1}$ & & & $\mathrm{C}_{2}$ \\
\hline S10 & $\mathrm{C}_{2}$ & & & $\mathrm{C}_{2}$ \\
\hline S11 & $\mathrm{C}_{1}$ & & & $\mathrm{C}_{1}$ \\
\hline $\mathrm{S} 12$ & $\mathrm{C}_{1}$ & & & $\mathrm{C}_{1}$ \\
\hline Stroke & \multicolumn{2}{|c|}{ Line } & \multicolumn{2}{|c|}{ Turn } \\
\hline Participants & $w_{1}$ & $w_{2}$ & $w_{1}$ & $w_{2}$ \\
\hline $\mathrm{P} 1$ & & $\mathrm{C}_{2}$ & & $\mathrm{C}_{2}$ \\
\hline $\mathrm{P} 2$ & $\mathrm{C}_{2}$ & & & $\mathrm{C}_{2}$ \\
\hline P3 & & $\mathrm{C}_{1}$ & & \\
\hline $\mathrm{P} 4$ & $\mathrm{C}_{1}$ & $\mathrm{C}_{2}$ & $\mathrm{C}_{1}$ & $\mathrm{C}_{2}$ \\
\hline P5 & $\mathrm{C}_{1}$ & $\mathrm{C}_{2}$ & & $\mathrm{C}_{1}$ \\
\hline P6 & & $\mathrm{C}_{1}$ & & \\
\hline P7 & $\mathrm{C}_{1}$ & $\mathrm{C}_{2}$ & $\mathrm{C}_{1}$ & \\
\hline P8 & & $\mathrm{C}_{1}$ & $\mathrm{C}_{1}$ & \\
\hline P9 & $\mathrm{C}_{1}$ & $\mathrm{C}_{2}$ & & \\
\hline P10 & $\mathrm{C}_{1}$ & & & $\mathrm{C}_{1}$ \\
\hline P11 & & & & \\
\hline P12 & & & & \\
\hline
\end{tabular}

$W_{1}$ and $W_{2}$ : First two (i.e. primary) common synergies.

$C_{i}$ : Indicates that the participant expressed $W_{1}$ or $W_{2}$ as their $i$-th individual synergy.

Empty cell: The participant did not express this common synergy as either their $1^{\text {st }}$ or $2^{\text {nd }}$ individual synergy. Grey cell: The participant did not complete the respective task. 
Table 4. Intrinsic Motivation Inventory (IMI) results.

\begin{tabular}{|c|c|c|c|c|}
\hline & Question & Stroke & Control & Difference \\
\hline 1. & $\begin{array}{l}\text { I think this activity could be beneficial to } \\
\text { me }\end{array}$ & $6.2 \pm 0.4$ & $3.6 \pm 0.7$ & $p=0.0043$ \\
\hline 2. & This activity required a lot of effort & $6.5 \pm 0.4$ & $4.6 \pm 0.7$ & $p=0.025$ \\
\hline 3. & I felt anxious while doing this activity & $2.7 \pm 0.7$ & $2.0 \pm 0.5$ & $p=0.38$ \\
\hline 4. & This activity did not keep my attention & $2.0 \pm 0.6$ & $1.6 \pm 0.3$ & $p=0.54$ \\
\hline 5. & I did this activity because I wanted to & $7.0 \pm 0.0$ & $6.6 \pm 0.3$ & $p=0.22$ \\
\hline 6. & I found this activity fun & $6.7 \pm 0.2$ & $6.4 \pm 0.4$ & $p=0.52$ \\
\hline 7. & This was an important activity to me & $6.8 \pm 0.2$ & $6.3 \pm 0.5$ & $p=0.39$ \\
\hline 8. & $\begin{array}{l}\text { I was satisfied with my performance at } \\
\text { this activity }\end{array}$ & $6.3 \pm 0.3$ & $6.1 \pm 0.3$ & $\mathrm{p}=0.85$ \\
\hline 9. & I was good at this activity & $5.5 \pm 0.4$ & $5.5 \pm 0.5$ & $\mathrm{p}=1.0$ \\
\hline 10. & $\begin{array}{l}\text { After a while, I became competent at this } \\
\text { activity }\end{array}$ & $5.8 \pm 0.4$ & $6.2 \pm 0.3$ & $\mathrm{p}=0.57$ \\
\hline
\end{tabular}



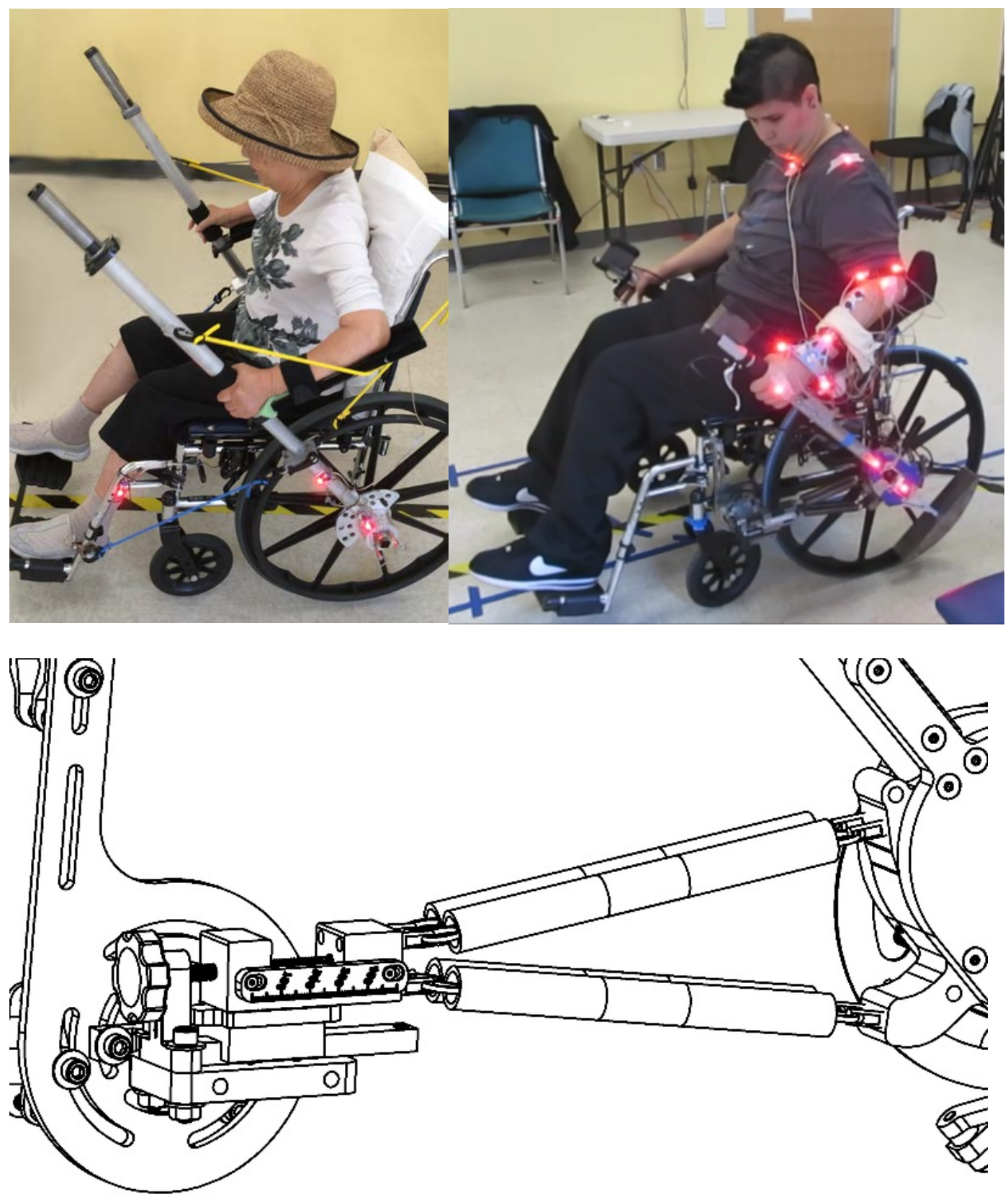

Figure 1. Top left: The lever-driven wheelchairs used in the first experiment. The arm supports and elastic bands position the paretic arm so the user can bimanually propel the chair. Top right. The improved lever-driven wheelchair used in the second experiment. The addition of handbrakes facilitated turning and straight ambulation. A clutch handle (back of gearbox) toggled the transmission between stationary and overground modes. Springs (both forward of the gearbox) serve as the elastic elements. Steel weights (top and bottom of lever) add inertia to enhance mechanical resonance. The experimental arrangement of motion capture markers (LED lights) and sEMG electrodes are also pictured. Bottom: Elastic elements and tensioning mechanism for the improved lever-driven wheelchair. The springs, although both forward of the gearbox, act in opposite rotational directions, the top springs resisting backward motion and the bottom springs resisting forward motion. This arrangement results in a highly linear angular stiffness coefficient over the lever's range of motion during operation. The adjustment knob increases the angular stiffness as it is tightened while retaining this linearity. 


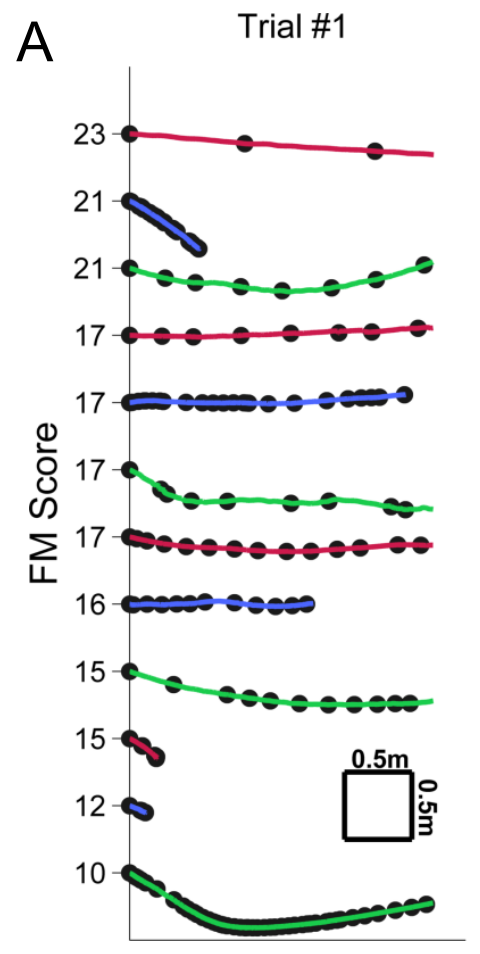

Trial \#10

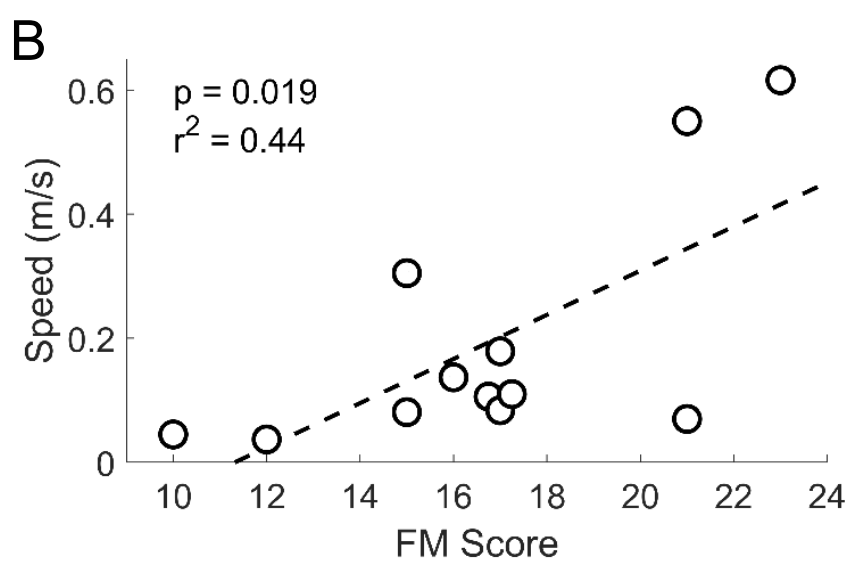

Figure 2. Overground trajectories of stroke participants during first experiment. A: Overhead view of the first and last trials of the 3-m driving task for all 12 subjects. Each line represents a different subject as they moved from left to right. The box at bottom left denotes the position scale. The starting y-position for each subject is offset according to their FM scores, with the lowest scores at the bottom and the highest scores at the top. The dots represent 2 -s intervals during the trial; thus, dots that are closer together indicate slower movement. We flipped the trajectories for the left-hemiparetic subjects so that the side of the wheelchair controlled by the impaired arm is always on the bottom side of the figure (i.e. the trajectories are presented as if all subjects had right hemiparesis). B: Regression of speed against FM showed a significant correlation. 


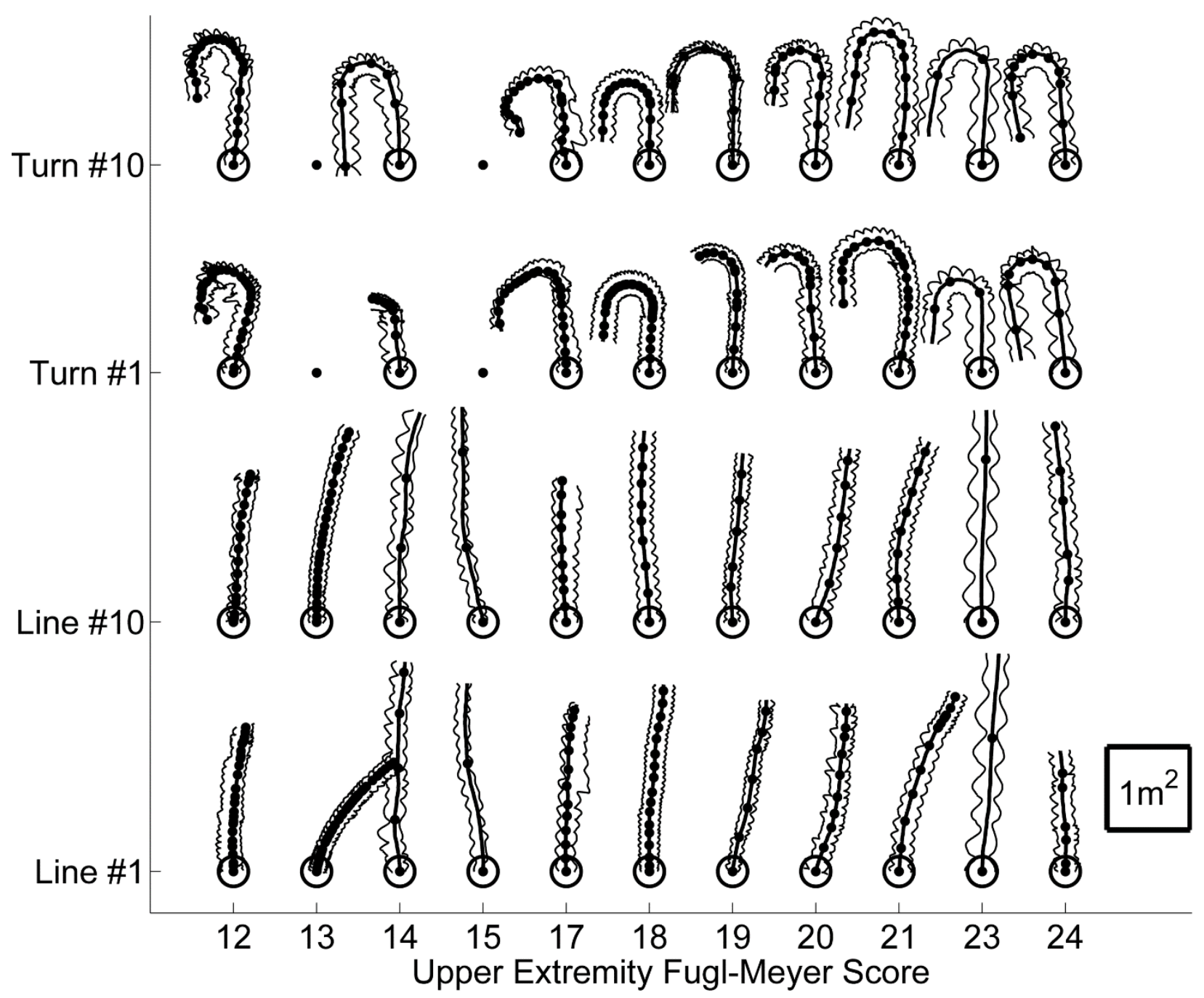

Figure 3. Overground trajectories of stroke participants during second experiment. Raw motion capture data for stroke participants. Each bold curve represents the trajectory traced by a participant during a single trial. The first and last trials from each condition (straight trajectory and $180^{\circ}$ turn) are plotted for each participant, and participants are ordered from left to right by FM score. Dots along the bold curve are spaced out at 2-s intervals providing a visualization of speed. The thin curves on immediately to the left and right of the bold curve represent the movement of the left and right levers respectively. When a lever is pulled back, the thin curve moves closer to the bold curve. When the lever is pushed forward, the thin curve moves away from the bold curve. In general, participants moved the levers in sync. Therefore, the space between the thin lines is narrow when both levers are pulled back, and wide when both levers are pushed forward. The data from participants with left-sided impairment have their data mirrored so that all trajectories are plotted as if the participant had right-sided impairment. 

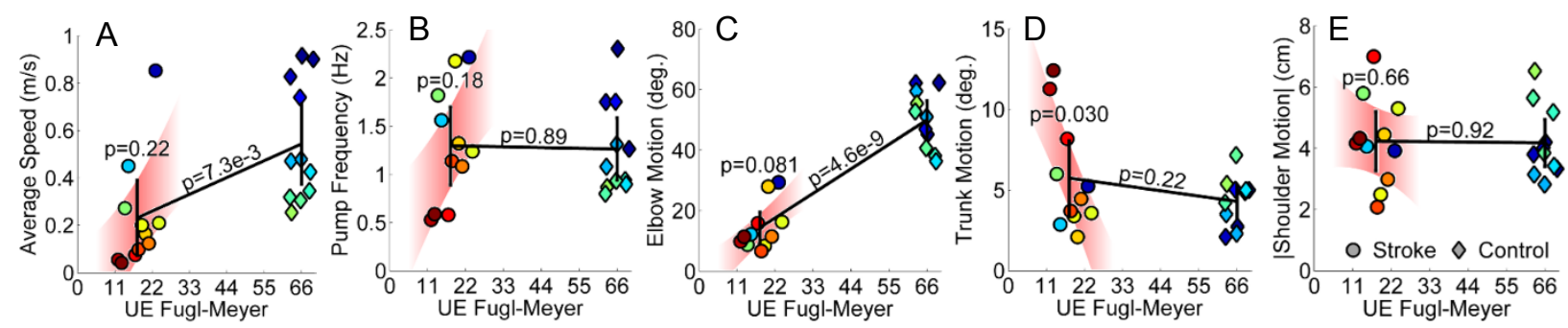

Figure 4. Straight-line trial kinematics. Each data point represents the value of a kinematic feature averaged over one participant. Solid lines and p-values indicate population differences between stroke participants and controls. Shaded bands depict $95 \%$ confidence regions of the mean trends across stroke participants between FM score and kinematic features. The associated p-values give the significance of the trends' slopes. The color of each data point indicates that participant's average speed, according to a heat map where red to blue represents slow to fast participants respectively. Control participant data points are arbitrarily scattered around a FM score of 66 to aid in visualization. A: Stroke participants were on average substantially slower than unimpaired controls. Although there was only a marginal correlation between FM score and speed, the slowest participants did have among the lowest FM scores. B: A wide range of pump frequencies was observed for both participant populations. Pump frequency was also only marginally correlated with FM score, but the slowest participants did pump particularly slowly. C: Elbow range of motion was substantially lower for stroke participants, and was weakly correlated with FM score, as might be expected. D: On average, stroke participants showed no increased trunk motion over controls. However, the three slowest participants exhibited larger trunk motion, suggesting the use of trunk movement as a compensatory strategy by the slowest participants. E: Shoulder movement differed minimally between stroke participants and controls, and was uncorrelated with FM score, evidence that shoulder movement was minimally involved in compensation. 

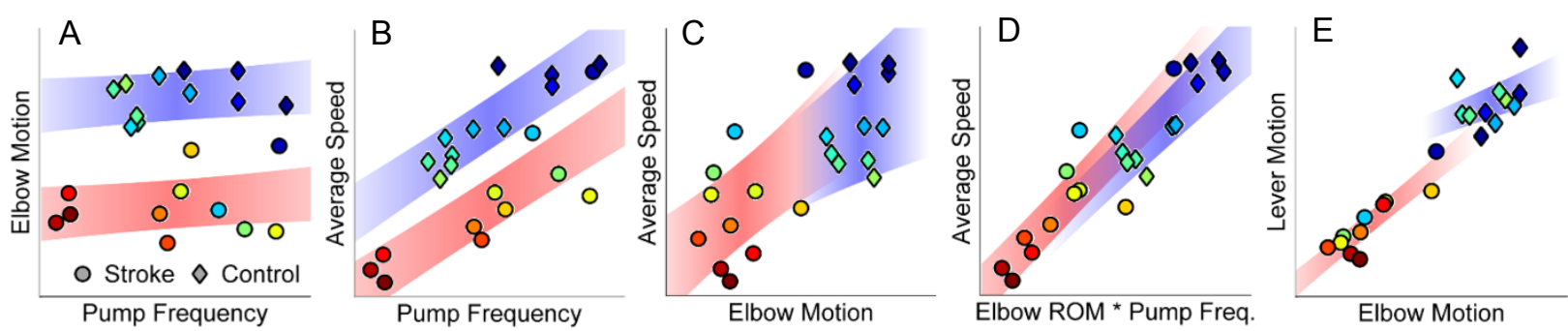

Figure 5. Straight-line trial kinematic regression analyses. Shaded regions represent $95 \%$ confidence regions for the trend lines for stroke participants (red) and controls (blue). That is, the trend line has a $95 \%$ chance of falling entirely within this region. Data point colors indicate that participant's average speed, with red to blue representing slowest to fastest. A: Elbow motion and pump frequency were uncorrelated with each other. B, C: Pump frequency and elbow range of motion were both correlated with average speed. D: When combined, these factors become strongly predictive of average speed. This implies that two uncorrelated motor capacities, the ability to quickly activate/deactivate muscles and the ability to extend the elbow, coordinate toward determining an individual's ambulation speed. E: Elbow motion was strongly correlated with lever motion for stroke participants, further supporting the conclusion that these participants moved the lever by extending their elbows, rather than through compensatory shoulder or trunk movement. 

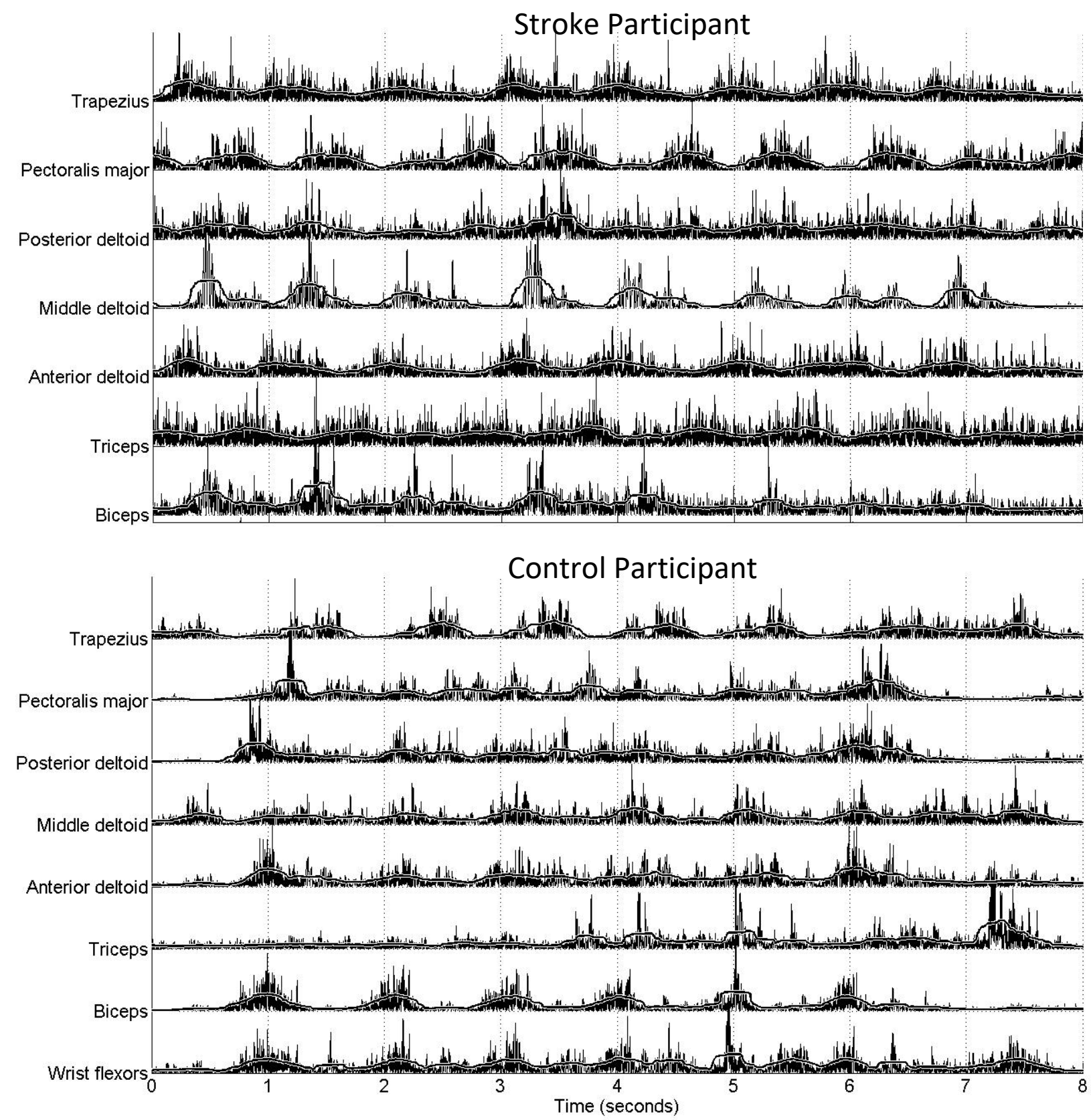

Figure 6. Raw EMG from chronic stroke and unimpaired participant. A $20-400 \mathrm{~Hz}$ bandpass filter was used to condition the signal and remove motion artifact, a $125 \mathrm{~ms}$ window moving RMS was used to estimate muscle activity. 

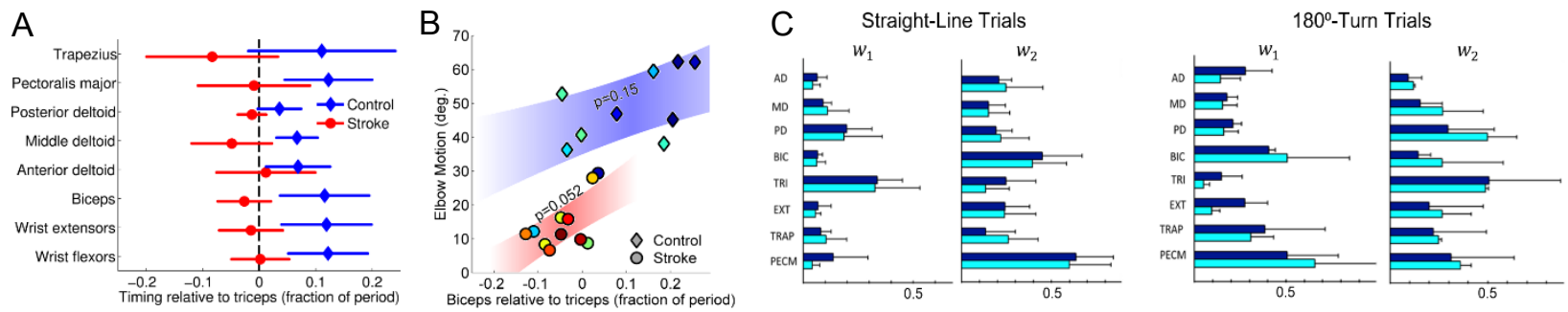

Figure 7. A: Muscle activation timing relative to triceps. Average activation timings of selected muscles relative to the activation of the triceps $($ at $t=0)$. Positive values indicate that the muscle was activated later than the triceps. Timing is measured in terms of fraction of that average period length during the trial. Unimpaired controls led with triceps activation, a pattern not maintained after stroke. Following stroke, the trapezius tends to lead the movement. B: Correlation of biceps-triceps timing with elbow movement. Chronic stroke participants' timing of the biceps relative to the triceps was correlated with the amplitude of their elbow movements. A similar but weaker correlation was found for unimpaired controls. This result is consistent with the theory that large elbow amplitudes require the triceps to extend the elbow during the arm movement, but that this extension requires the triceps to contract without co-contraction of the biceps. C: The common muscle synergies $w_{1}$ and $w_{2}$, for each experimental task. Bars indicate the mean normalized activation of each muscle involved in the synergy across subjects who expressed that synergy. Error bars indicate the standard deviation about these means. Synergies of stroke participants are shown in cyan, and synergies of control participants in blue 